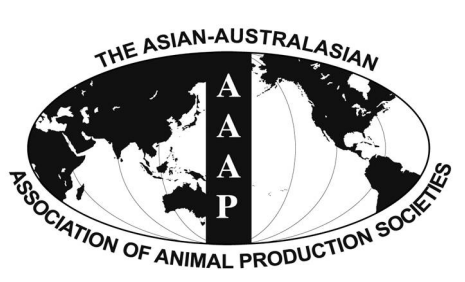

Open Access

Asian Australas. J. Anim. Sci.

Vol. 28, No. 12 : 1703-1712 December 2015

http://dx.doi.org/10.5713/ajas.15.0051

www.ajas.info

pISSN 1011-2367 elSSN 1976-5517

\title{
Subcellular Characterization of Porcine Oocytes with Different Glucose-6-phosphate Dehydrogenase Activities
}

\author{
Bo Fu ${ }^{1,2, a}$, Liang Ren ${ }^{3, a}$, Di Liu ${ }^{2,3, *}$, Jian-Zhang Ma ${ }^{1}$, Tie-Zhu An ${ }^{1}$, Xiu-Qin Yang ${ }^{3}$, Hong Ma ${ }^{2}$, \\ Dong-Jie Zhang ${ }^{2}$, Zhen-Hua Guo ${ }^{2}$, Yun-Yun Guo ${ }^{3}$, Meng Zhu ${ }^{3}$, and Jing Bai ${ }^{4}$ \\ ${ }^{1}$ Heilongjiang Academy of Agricultural Sciences Postdoctoral Programme, \\ Northeast Forestry University Postdoctoral Programme, Harbin 150086, China
}

\begin{abstract}
The in vitro maturation (IVM) efficiency of porcine embryos is still low because of poor oocyte quality. Although brilliant cresyl blue positive $(\mathrm{BCB}+)$ oocytes with low glucose-6-phosphate dehydrogenase $(\mathrm{G} 6 \mathrm{PDH})$ activity have shown superior quality than BCB negative (-) oocytes with high G6PDH activity, the use of a BCB staining test before IVM is still controversial. This study aimed to shed more light on the subcellular characteristics of porcine oocytes after selection using BCB staining. We assessed germinal vesicle chromatin configuration, cortical granule (CG) migration, mitochondrial distribution, the levels of acetylated lysine 9 of histone $\mathrm{H} 3$ (AcH3K9) and nuclear apoptosis features to investigate the correlation between G6PDH activity and these developmentally related features. A pattern of chromatin surrounding the nucleoli was seen in $53.0 \%$ of $\mathrm{BCB}+$ oocytes and $77.6 \%$ of $\mathrm{BCB}+$ oocytes showed peripherally distributed CGs. After IVM, 48.7\% of $\mathrm{BCB}+$ oocytes had a diffused mitochondrial distribution pattern. However, there were no significant differences in the levels of AcH3K9 in the nuclei of blastocysts derived from $\mathrm{BCB}+$ and BCB- oocytes; at the same time, we observed a similar incidence of apoptosis in the $\mathrm{BCB}+$ and control groups. Although this study indicated that G6PDH activity in porcine oocytes was correlated with several subcellular characteristics such as germinal vesicle chromatin configuration, CG migration and mitochondrial distribution, other features such as AcH3K9 level and nuclear apoptotic features were not associated with G6PDH activity and did not validate the BCB staining test. In using this test for selecting porcine oocytes, subcellular characteristics such as the AcH3K9 level and apoptotic nuclear features should also be considered. Adding histone deacetylase inhibitors or apoptosis inhibitors into the culture medium used might improve the efficiency of IVM of BCB+ oocytes. (Key Words: Porcine Oocytes, Brilliant Cresyl Blue, Embryonic Development, Subcellular Characteristics)
\end{abstract}

\section{INTRODUCTION}

Glucose-6-phosphate dehydrogenase (G6PDH) activity decreases with oocyte maturation (Alm et al., 2005;

\footnotetext{
* Corresponding Author: Di Liu. Tel: +86-045186657928, Fax:+86-045186657928, E-mail: liudi1963616@163.com

${ }^{2}$ Institute of Animal Husbandry Research, Heilongjiang Academy of Agricultural Sciences, Harbin 150086, China.

${ }^{3}$ College of Animal Science, Northeast Agriculture University, Harbin 150030, China.

4 Modern Education Technology and Information Center, Heilongjiang University of Chinese Medicine, Harbin 150040, China.

${ }^{\text {a }}$ These two authors contributed equally to this work.

Submitted Jan. 18, 2015; Revised Apr. 11, 2015; Accepted May 8, 2015
}

Bhojwani et al., 2007). A brilliant cresyl blue (BCB) staining test can show different intracellular activity of G6PDH in oocytes because the G6PDH enzyme converts $\mathrm{BCB}$ from blue to a colorless state. Those oocytes still undergoing growth have high levels of $\mathrm{G} 6 \mathrm{PDH}$, so $\mathrm{BCB}$ is made colorless while fully-grown oocytes with superior developmental competence have a low amount of G6PDH, and BCB remains blue (Tiffin et al., 1991). Thus, the G6PDH level is an indirect marker of oocyte growth. Therefore, compared with conventional morphological selection methods, BCB staining seems to be a reliable test for selecting mature porcine oocytes. Earlier studies have shown that this test can select oocytes with superior quality in some species such as the pig, goat, bovine, mouse, and dog in terms of nuclear maturation, cleavage rate and 
blastocyst yield (Ericsson et al., 1993; Rodríguez-González et al., 2003; Wu et al., 2007; Rodrigues et al., 2009; Mirshamsi et al., 2013). However, the predictive value of the BCB staining test is still controversial (Kempisty et al., 2011; Pawlak et al., 2011a; Opiela and KatskaKsiazkiewicz, 2013; Pereira et al., 2014; Pawlak et al., 2014). For example, oocytes derived from prepubertal animals performed poorly even though these oocytes were $\mathrm{BCB}+$; only $12 \%$ and $4 \%$ of $\mathrm{BCB}+$ oocytes derived from prepubertal heifers and prepubertal goats formed blastocysts, respectively (Rodríguez-González et al., 2002; Pujol et al., 2004). This suggests that not all $\mathrm{BCB}+$ oocytes constitute a homogeneous population in terms of developmental competence.

Chromatin configuration is correlated with oocyte growth and the distributions of organelles such as cortical granules (CGs) and mitochondria are also objective indicators of cytoplasmic maturation. The levels of acetylated lysine 9 of histone $\mathrm{H} 3$ (AcH3K9) and apoptosis reflect the developmental potential of embryos. Date are scarce concerning these subcellular characteristics in porcine oocytes after selecting with $\mathrm{BCB}$ staining, so the significance of these features among $\mathrm{BCB}+, \mathrm{BCB}-$, and control oocytes should be clarified. We investigated several such factors including germinal vesicle (GV) chromatin configuration, $\mathrm{CG}$ migration, mitochondrial distribution, AcH3K9 levels, and apoptotic nuclear features of $\mathrm{BCB}+$, $\mathrm{BCB}-$, and control oocytes. These studies should clearly characterize $\mathrm{BCB}+, \mathrm{BCB}-$, and control porcine oocytes in terms of organelle features and contribute to the establishment of better in vitro maturation (IVM) systems for porcine $\mathrm{BCB}+$ oocytes.

\section{MATERIALS AND METHODS}

\section{Chemicals}

All chemicals were purchased from Sigma-Aldrich (St. Louis, MO, USA) unless otherwise indicated.

\section{Brilliant cresyl blue staining test}

Porcine ovaries were collected at a local abattoir and transported to the laboratory in $0.9 \% \mathrm{NaCl}$ solution at $35^{\circ} \mathrm{C}$. Cumulus-oocyte complexes (COCs) were aspirated from 3 to $6 \mathrm{~mm}$ diameter antral follicles by using a $20 \mathrm{~mL}$ disposable syringe with an 18-gauge needle. After being collected, COCs were washed three times in Dulbecco's phosphate buffered saline (DPBS) supplemented with 13 $\mu \mathrm{mol} / \mathrm{L}$ of $\mathrm{BCB}$ and exposed to $13 \mu \mathrm{mol} / \mathrm{L}$ of $\mathrm{BCB}$ diluted in DPBS for $90 \mathrm{~min}$ at $38.5^{\circ} \mathrm{C}$ in a humidified air atmosphere. Following BCB exposure, the COCs were then transferred to DPBS and washed twice. COCs were then examined under a stereomicroscope and divided into two groups: $\mathrm{BCB}+$ (coloured cytoplasm, low G6PDH) and $\mathrm{BCB}-$ (colourless cytoplasm, increased G6PDH), respectively. $\mathrm{COCs}$ untreated with $\mathrm{BCB}$ were used as control.

\section{Observation of germinal vesicle chromatin configuration}

COCs were denuded of cumulus cells by vortexing in the presence of $0.1 \%$ hyaluronidase in DPBS. The denuded oocytes were cultured in tissue culture medium (TCM) 199 containing $10 \mu \mathrm{g} / \mathrm{mL}$ Hoechst 33,342 for $30 \mathrm{~min}$ in humidified air. Oocytes of the same group were then placed on a glass slide and squashed with coverslips to visualize the GV. The GVs were examined by fluorescence optics. The GV chromatin configuration of oocytes was classified into the non-surrounded nucleoli (NSN) pattern with diffuse chromatin throughout the nucleus and the surrounded nucleoli (SN) pattern with chromatin condensed into a perinucleolar rim.

\section{In vitro maturation of oocytes}

After being selected with BCB staining, COCs with an evenly distributed cytoplasm and at least three compact layers of cumulus cells were selected and washed three times in TCM 199 enriched with 10\% (v/v) fetal bovine serum, and then were washed twice with the maturation medium (TCM 199 supplemented with $0.1 \%$ poly vinyl alcohol (PVA), $3.05 \mathrm{mM}$ D-glucose, $0.91 \mathrm{mM}$ sodium pyruvate, $0.57 \mathrm{mM}$ cysteine, $10 \mathrm{IU} / \mathrm{mL}$ human chorionic gonadotrop, $10 \mathrm{IU} / \mathrm{mL}$ pregnant mare serum gonadotropin, $10 \mathrm{ng} / \mathrm{mL}$ epidermal growth factor, $10 \%$ porcine follicular fluid, $75 \mu \mathrm{g} / \mathrm{mL}$ penicillin $\mathrm{G}$, and $50 \mu \mathrm{g} / \mathrm{mL}$ streptomycin). After about $21 \mathrm{~h}$ of culture with maturation medium, oocytes were cultured without hormones for another $21 \mathrm{~h}$ at $38.5^{\circ} \mathrm{C}, 5 \% \mathrm{CO}_{2}$. After IVM, COCs from each $\mathrm{BCB}+$, $\mathrm{BCB}-$, and control group were freed from surrounding cumulus cells and oocytes with the first polar body (finished nuclear maturation) were evaluated.

\section{Evaluation of cortical granules migration in porcine oocytes}

After denuded of cumulus cells, oocytes were fixed with $4 \%(\mathrm{w} / \mathrm{v})$ paraformaldehyde in PBS for $30 \mathrm{~min}$ at $20^{\circ} \mathrm{C}$. After fixation, oocytes were rinsed with blocking solution (PBS containing $0.3 \% \mathrm{BSA}$ and $100 \mathrm{mM}$ glycine and 100 $\mathrm{mM}$ glycine) for $5 \mathrm{~min}$, then were permeabilized with $0.1 \%$ Triton X-100 in PBS for $5 \mathrm{~min}$. After being washed twice in blocking solution, the oocytes were then labeled with 100 $\mathrm{mg} / \mathrm{mL}$ fluorescein isothiocyanate-labeled peanut agglutinin in PBS for $30 \mathrm{~min}$ in a dark box. Finally, the oocytes were rinsed thoroughly with PBS-PVA. The oocytes from each sample were mounted between a slide and coverslip, then 
scanned and recorded with confocal fluorescent microscopy.

\section{Evaluation of mitochondrion distribution in porcine} oocytes

After maturation, oocytes were transferred into denuding medium with hyaluronidase in a $1.5-\mathrm{mL}$ centrifuge tube. The oocytes were vigorously vortexed for 4 to $5 \mathrm{~min}$, then transferred into manipulation medium. Oocytes with an intact plasma membrane and the first polar body were stained with Mito-Tracker Red CMXRos (Invitrogen, Eugene, Oregon, USA). The dye was used at a concentration of $250 \mathrm{nM}$ in TCM 199 for $30 \mathrm{~min}$ at $38.5^{\circ} \mathrm{C}$. After staining, oocytes were briefly rinsed in DPBS and fixed in $4 \%$ paraformaldehyde in DPBS for $30 \mathrm{~min}$ at $38.5^{\circ} \mathrm{C}$. The oocytes from each sample were mounted between a slide and coverslip, then scanned and recorded with confocal fluorescent microscopy. With regard to mitochondrion distribution, oocytes with diffuse mitochondrion throughout the entire cytoplasm were classified as mature and those with peripheral and semiperipheral mitochondrion as immature.

\section{Production of parthenogenetic activation embryos}

Only denuded oocytes with the first polar body were selected. For activation, $1 \mathrm{DC}$ pulse of $2.0 \mathrm{kV} / \mathrm{cm}$ for $30 \mu \mathrm{s}$ was applied, by using a BTX-Cell Manipulator 2001 (Genetronics, San Diego, CA, USA). The medium used for activation was $0.3 \mathrm{M}$ mannitol supplemented with $0.1 \%$ PVA, $0.1 \mathrm{mM} \mathrm{CaCl}$, $0.1 \mathrm{mM} \mathrm{MgCl}_{2}$ and $0.5 \mathrm{mM}$ Hepes. Then embryos were treated with $2 \mathrm{mM}$ 6-DMAP (4dimethylaminopyridine) for $3 \mathrm{~h}$. Activated embryos were cultured in PZM-3 medium then examined for cleavage at $44 \mathrm{~h}$ after activation. The percentage of blastocyst derived from $\mathrm{BCB}+, \mathrm{BCB}_{-}-$, and control oocytes were determined on day 7.

\section{Global acetylation levels of histone $\mathrm{H3}$ at lysine 9 Assays}

For day 7 embryos derived from parthenogenetically activated (PA), zonae pellucidae were removed by pronase treatment. Blastocysts were washed in PBS, then fixed for $15 \mathrm{~min}$ in $4 \%$ paraformaldehyde in PBS, and embryos were permeabilized with $0.2 \%$ Triton X-100 in PBS-PVA for 30 min. Samples were blocked overnight at $4^{\circ} \mathrm{C}$ in blocking solution. The samples were incubated with a rabbit polyclonal antibody to AcH3K9 (Millipore, Darmstadt, Germany ) diluted 1:200 for $12 \mathrm{~h}$ at $4^{\circ} \mathrm{C}$. After extensive washing, samples were incubated with a secondary antibody of Alexa Fluor 594-labeled goat anti-rabbit IgG (Invitrogen, Eugene, Oregon, USA) for AcH3K9. After washing three times, blastocysts were stained with Hoechst 33342 and mounted on slides. Fluorescence was observed under a fluorescence digital microscope (Axiovert 200M, ZEISS,
Shanghai, China), and the fluorescence intensity of each pixel was transformed to obtain a quantitative measure using Image-J software. Quantification of AcH3K9 signal intensities in $\mathrm{BCB}+$ and $\mathrm{BCB}-$ groups was expressed relative to that of the control embryos (set as 100\%). Quantification of the AcH3K9 is represented as the mean \pm standard error (SE). Values with different superscripts means significant difference $(p<0.05)$.

\section{Apoptosis assays}

Apoptosis assays were carried out using a DeadEnd Fluorometric TUNEL System (Promega, Madison, WI, USA). After being washed and fixed, the blastocyst was permeabilized for staining. After equilibration, embryos were incubated with terminal deoxynucleotidyl transferase nick-end labeling (TUNEL) reaction mixture in the dark box for $1 \mathrm{~h}$ at $37^{\circ} \mathrm{C}$. The reaction was terminated in $2 \times \mathrm{SSC}$ for $15 \mathrm{~min}$. Finally, the DNA was stained with Hoechst 33342. Samples were mounted on glass slides for apoptosis assays. Blastocysts that served as a positive control were incubated in DNase I solutions ( $5 \mathrm{U} / 50 \mu \mathrm{L})$.

\section{Statistical analysis}

For each treatment, three replicates were run. All data were subjected to one-way analysis of variance by using SPSS ver. 16.0 (SPSS Inc., Chicago, IL, USA). Differences were considered significant at a level of $p<0.05$. Results were presented as mean $\pm \mathrm{SE}$.

\section{RESULTS}

\section{Correlation of G6PDH activity with germinal vesicle stage chromatin configurations}

Following BCB exposure, the oocytes were examined under a stereomicroscope and classed as $\mathrm{BCB}+$ (blue cytoplasm) and BCB-(colorless cytoplasm) (Figure 1).

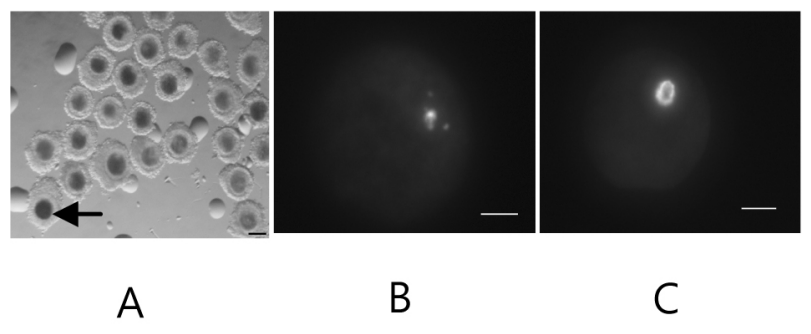

Figure 1. Cumulus-oocyte complexes after brilliant cresyl blue (BCB) staining and germinal vesicle (GV) chromatin configurations of porcine oocytes. (A) The arrow indicates $\mathrm{BCB}+$ oocyte with blue ooplasm, whereas colorless ooplasm indicates $\mathrm{BCB}$ - oocyte. $\mathrm{Bar}=100 \mu \mathrm{m}$. The GV chromatin configuration of oocytes was stained by $10 \mu \mathrm{g} / \mathrm{mL}$ Hoechst 33342. (B) Nonsurrounded nucleoli (NSN) pattern with diffuse chromatin throughout the nucleus. (C) Surrounded nucleoli (SN) pattern with chromatin condensed into a perinucleolar rim. Bar $=20 \mu \mathrm{m}$. 


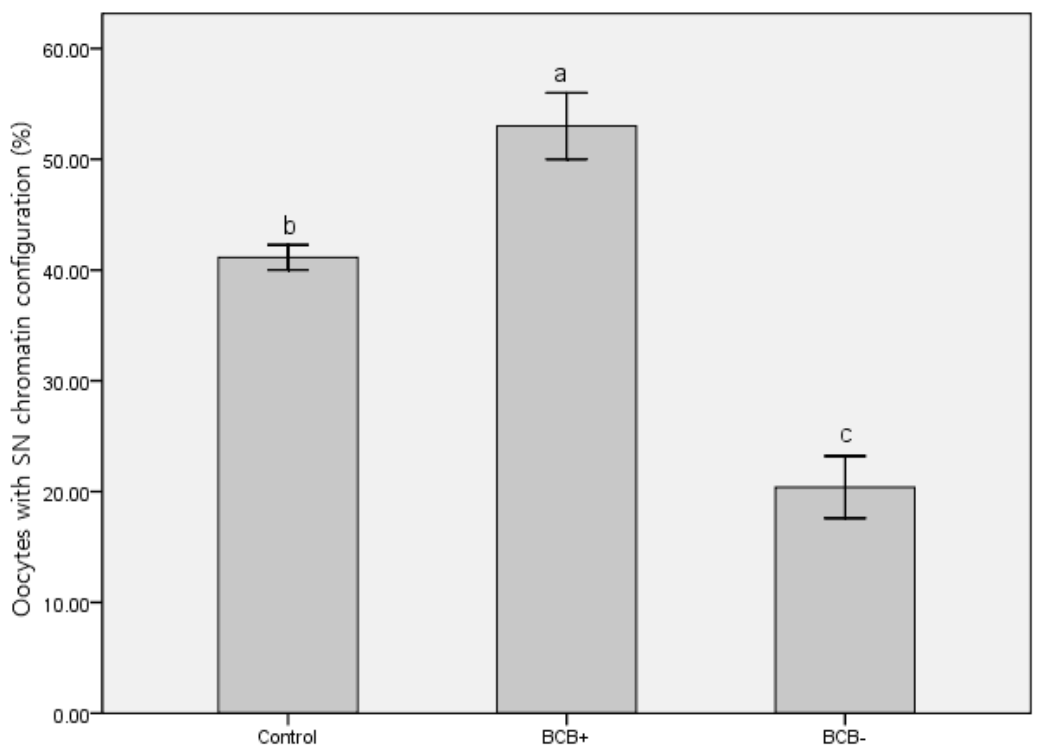

Figure 2. Germinal vesicle chromatin configurations of brilliant cresyl blue positive $(\mathrm{BCB}+)$, $\mathrm{BCB}-$, and control oocytes. Different superscripts show statistically significant differences between oocytes from $\mathrm{BCB}+, \mathrm{BCB}-$, and control groups $(\mathrm{p}<0.05)$. The experiment was repeated for three times. SN, surrounded nucleoli.

Figure 1 also shows two kinds of chromatin configuration we defined as SN and NSN. While most of the BCBoocytes had an NSN configuration, more BCB+ oocytes were of the SN pattern (Figure 2). This result suggests that chromatin configuration patterns were associated with G6PDH activity in oocytes.

\section{Effect of G6PDH activity on meiotic competence}

After $42 \mathrm{~h}$ in IVM medium, oocytes were denuded of cumulus cells. Only those oocytes with the first polar body extruded were regarded as having finished nuclear maturation (Figure 3). In total, $236 \mathrm{BCB}+, 331 \mathrm{BCB}-$, and 303 control oocytes were used to determine nuclear status. Significantly more $\mathrm{BCB}+$ oocytes had finished nuclear maturation than in the $\mathrm{BCB}-$ and control groups $(\mathrm{p}<0.05)$, with $\mathrm{BCB}$ - oocytes having the lowest percentage of nuclear maturation (Figure 4). This suggests that G6PDH activity

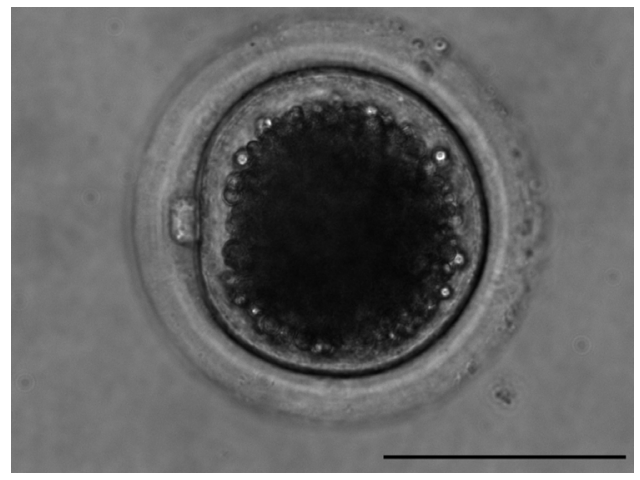

Figure 3. The mature oocyte with the first polar. Cumulus-oocyte complexes were freed from surrounding cumulus cells and oocytes with the first polar body (at MII) were evaluated. Bar = $100 \mu \mathrm{m}$. was inversely associated with maturation of porcine oocytes.

\section{Correlation of G6PDH activity with cortical granule migration}

CGs migration patterns in $\mathrm{BCB}+, \mathrm{BCB}^{-}$, and control oocytes were evaluated under a confocal microscope. Figure 5 shows the two categories of CG migration patterns. Oocytes with CGs arranged throughout the entire cytoplasm were classified as immature and those with peripheral CGs as mature. A peripheral distribution was significantly more prevalent in $\mathrm{BCB}+$ oocytes than in $\mathrm{BCB}-$ and control oocytes $(p<0.05)$ (Figure 6$)$. Thus, there was a negative association between G6PDH activity and the incidence of mature oocytes with peripheral CGs.

\section{Association of G6PDH activity with mitochondrial distribution}

The mitochondrial distributions in $\mathrm{BCB}+, \mathrm{BCB}^{-}$, and control oocytes were evaluated under a confocal microscope. Figure 7 shows the three categories of distribution. The peripheral pattern showed fluorescent signals distributed in the periphery of the oocyte; the semiperipheral pattern showed fluorescent signals covering most of the cytoplasmic volume without its central part; the diffuse pattern showed mitochondria distributed homogeneously throughout the ooplasm. Oocytes with the diffuse pattern were classified as mature and those with peripheral and semiperipheral mitochondrial distributions as immature. As expected, a higher proportion of oocytes with the diffuse pattern were found in BCB+ oocytes after IVM $(\mathrm{p}<0.05)$ (Figure 8). 


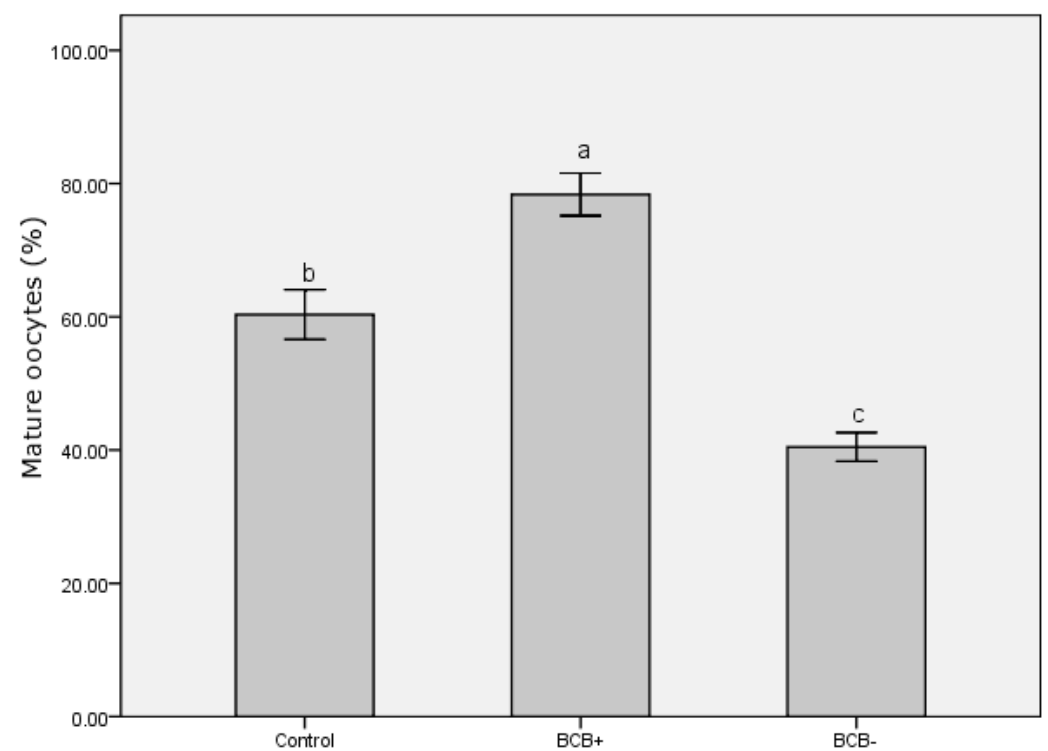

Figure 4. Nuclear maturation of brilliant cresyl blue positive $(\mathrm{BCB}+), \mathrm{BCB}-$, and control oocytes. Different superscripts show statistically significant differences between oocytes from $\mathrm{BCB}+, \mathrm{BCB}-$, and control groups $(\mathrm{p}<0.05)$. The experiment was repeated for three times.

Effect of G6PDH activity on the development of parthenogenetically activated embryos

$\mathrm{PA}$ embryos derived from $\mathrm{BCB}+, \mathrm{BCB}^{-}$, and control oocytes were examined for cleavage at $48 \mathrm{~h}$ after activation. The percentages of blastocysts derived from $\mathrm{BCB}+, \mathrm{BCB}-$, and control oocytes were determined on day 7. Regarding the rate of cleavage, there were no significant differences among the $\mathrm{BCB}+, \mathrm{BCB}-$, and control groups. A significantly higher fraction of $\mathrm{BCB}+$ oocytes developed into blastocysts compared with $\mathrm{BCB}-$ oocytes $(\mathrm{p}<0.05)$ whereas no significant difference was observed between the $\mathrm{BCB}+$ and control groups (Table 1).

Detection of histone acetylation in blastocysts derived from $\mathrm{BCB}+$ or $\mathrm{BCB}-$ oocytes

No signals could be detected in blastocysts without

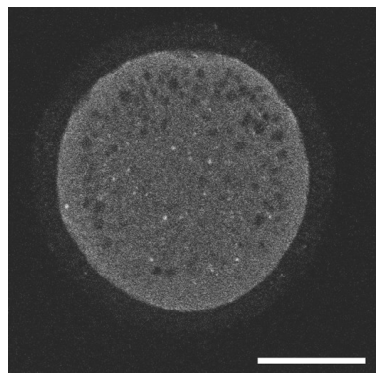

A

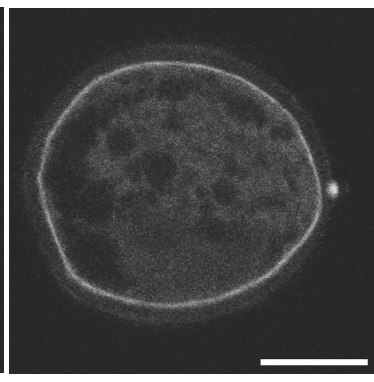

B
Figure 5. The confocal micrographs of cortical granules (CGs) migration in porcine oocytes. (A) CGs were distributed in the whole oocyte cytoplasma including the cortex. (B) CGs were distributed at the cortex and closely located beneath the membrane and formed a brilliant continuous ring. Bar $=50 \mu \mathrm{m}$. using primary or secondary antibodies, indicating specificity of staining by the primary antibody (data not shown). The global AcH3K9 in the nuclei of blastomeres derived from $\mathrm{BCB}+$ and $\mathrm{BCB}-$ oocytes were compared, but no significant difference was observed (Figure 9).

Apoptotic index of parthenogenetically activated blastocysts derived from $\mathrm{BCB}+, \mathrm{BCB}-$ and control oocytes

$\mathrm{BCB}+, \mathrm{BCB}^{-}$, and control groups were subjected to TUNEL analysis because the apoptotic index is another criterion for blastocyst quality. A blastocyst was scored as TUNEL positive when distinct, green fluorescence signals were observed. Although the $\mathrm{BCB}-$ group showed a higher apoptotic index, we observed a similar low incidence of apoptosis in $\mathrm{BCB}+$ and control groups (Figure 10).

\section{DISCUSSION}

The BCB staining test is claimed to be an efficient method to select porcine oocytes of high quality. However, several studies have questioned the reliability of BCB staining test for routine IVM. The lack of significant differences in blastocyst formation rates between $\mathrm{BCB}+$ and control groups led Opiela et al. (2008) to consider the BCB staining test questionable. Pawlak et al. (2014) found a high incidence of apoptosis and major variations in the diameter of $\mathrm{BCB}+$ oocytes, making the $\mathrm{BCB}$ staining test a less effective selection tool than reported previously. Moreover, the $\mathrm{BCB}+$ oocytes derived from pre-pubertal females and cycling gilts also differed in many aspects (Pawlak et al., 2011a,b). Overall, the quality of oocytes is a complex trait, 


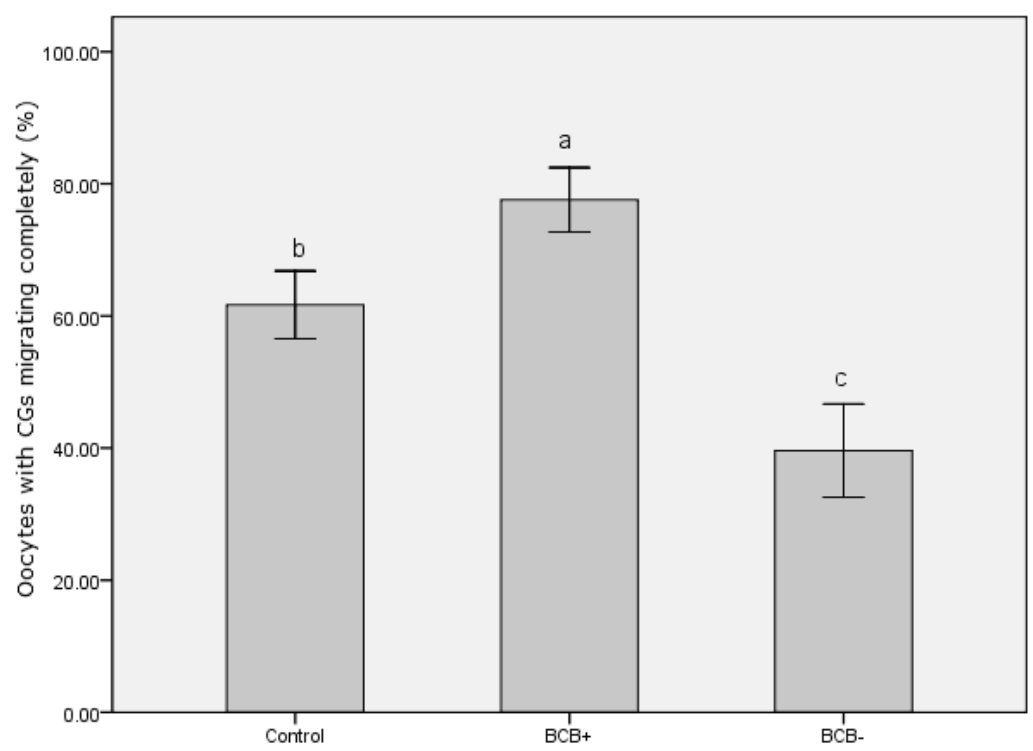

Figure 6. Cortical granules (CGs) migration of brilliant cresyl blue positive (BCB+), $\mathrm{BCB}-$, control oocytes. Different superscripts show statistically significant differences between oocytes from $\mathrm{BCB}+, \mathrm{BCB}-$, and control groups $(\mathrm{p}<0.05)$. The experiment was repeated for three times.

and these ambivalent phenomena might arise from heterogeneity in $\mathrm{BCB}+$ porcine oocytes. We need to evaluate the relationship between G6PDH activity and several subcellular characteristics in porcine oocytes after $\mathrm{BCB}$ staining selection to identify such heterogeneity. Here we investigated several subcellular characteristics such as GV chromatin configuration, CG migration, mitochondrial distribution, AcH3K9 level, and apoptotic nuclear features after BCB staining.

Chromatin condensation is associated with oocyte maturation and highly competent porcine oocytes have a more condensed chromatin configuration (Sun et al., 2004). Moreover, mouse oocytes with an SN configuration were more competent for development than oocytes with an NSN pattern (Wu et al., 2007). This suggests that the SN configuration in oocytes represents a stage toward ovulation and completion of oocyte growth (Guthrie and Garrett,

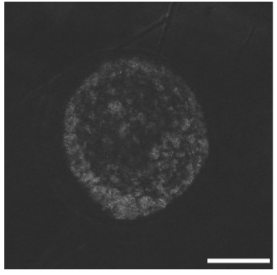

A

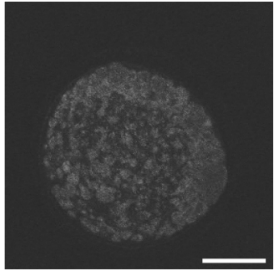

B

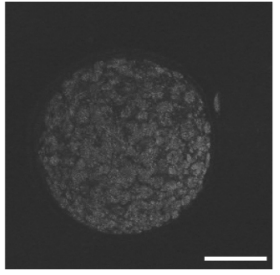

C
Figure 7. The confocal micrographs of mitochondrion distribution in porcine oocytes. (A) Fluorescent signals distributed in the peripheral (peripheral pattern). (B) Fluorescent signals covering most of the cytoplasmic volume without its central part (semiperipheral pattern). (C) Mitochondria distributed homogeneously throughout the ooplasm (diffuse pattern). Bar $=$ $50 \mu \mathrm{m}$.
2000). In the present study, the percentage of the SN pattern in $\mathrm{BCB}+$ oocytes was significantly higher than in control and $\mathrm{BCB}-$ oocytes. This indicated that $\mathrm{BCB}+$ oocytes might have higher developmental potential in terms of chromatin configuration.

Organelle distributions are also objective indicators of cytoplasmic maturation in oocytes. During maturation, the migration of CGs to the cortex relies on microfilament assembly, and a peripheral CG distribution is one indication of oocyte maturity. We found an increased rate of cytoplasm maturation for the $\mathrm{BCB}+$ group $(77.6 \%)$ characterized by peripheral CG migration compared with the control groups $(61.7 \%)$ and the BCB-groups (39.6\%). Delays in CG migration during maturation could be caused by an intrinsic deficiency in BCB- oocytes (Silva et al., 2013). Unlike CGs, mitochondria are mostly concentrated in the periphery of the oocyte at the GV stage, indicating contact with cumulus cell cytoplasmic projections. They then migrate toward the center during maturation (Sun et al., 2001). A homogeneous distribution of mitochondria in the cytoplasm is positively associated with adenosine triphosphate content and reflects changes in energy requirement accompanying cytoplasmic maturation, which directly influences fertilization outcomes in the pig (Yang et al., 2010). Our finding that the rate of $\mathrm{BCB}+$ oocytes with a diffuse mitochondrial distribution was significantly higher than in the other groups also indicated a higher level of cytoplasmic maturation and stronger developmental potential.

We did not find a significant difference between the blastocyst formation rates of $\mathrm{BCB}+$ and the control group in this study. This result is consistent with other reports (Opiela et al., 2008; Ishizaki et al., 2009) and might have 


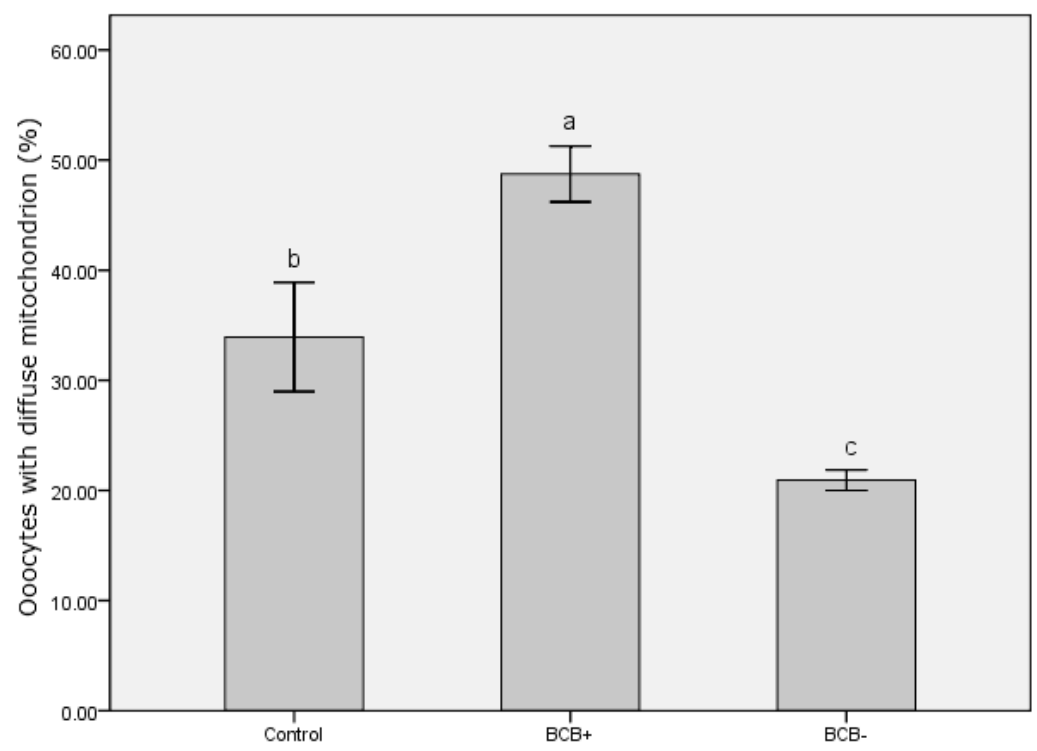

Figure 8. Mitochondrion distribution of brilliant cresyl blue positive $(\mathrm{BCB}+), \mathrm{BCB}-$, and control oocytes. Different superscripts show statistically significant differences between oocytes from $\mathrm{BCB}+, \mathrm{BCB}-$, and control groups $(\mathrm{p}<0.05)$. The experiment was repeated for three times.

resulted from a prolonged incubation time during $\mathrm{BCB}$ staining. In addition, porcine oocytes are commonly recovered from the ovaries of slaughtered animals and oocytes may be at various stages of the estrous cycle. Thus, $\mathrm{BCB}+$ oocytes derived from different stages of the estrous cycle (e.g., pre-pubertal females or cycling gilts) still differ in many aspects; oocytes from cycling gilts have higher developmental competence than those from pre-pubertal pigs, even though $\mathrm{BCB}+$ oocytes predominated among the oocytes collected from both groups (Pawlak et al., 2011a). Thus, the heterogeneity of $\mathrm{BCB}+$ porcine oocytes and a lack of significant difference between the blastocyst formation rates of $\mathrm{BCB}+$ and control groups decreases the validity of the $\mathrm{BCB}$ staining test.

The AcH3K9 level and apoptotic nuclear features affect the developmental potential of embryos and the different G6PDH activities as measured by BCB staining could not be reflected by these two subcellular characteristics in this study. Acetylation levels of nuclear histones are major epigenetic modifications of the genome, which play important roles in the development of embryos (Dai et al., 2010; Das et al., 2010). To our knowledge, the effects of selecting porcine oocytes using the $\mathrm{BCB}$ staining test on histone modifications have not yet been investigated. The global acetylation levels of $\mathrm{H} 3 \mathrm{~K} 9$ were analyzed here. We found that the $\mathrm{BCB}+$ and $\mathrm{BCB}-$ groups showed similar global levels of AcH3K9 at the blastocyst stage. This result is in agreement with an earlier report in cattle ( $\mathrm{Su}$ et al., 2012). Given the similar acetylation levels of H3K9 between groups, the $\mathrm{BCB}$ staining test seems not to be valuable for evaluating oocyte quality for this factor. More studies measuring the acetylation level of other histone lysine residues would be helpful to verify BCB staining test further.

Although the level of apoptosis is another criterion of embryo quality, the value of measuring G6PDH activity as an indirect marker of apoptosis seems to be questionable. The lack of significant differences between the ratio of densitometric signals of $\mathrm{Bax}$ to $\mathrm{Bcl}-2$ in $\mathrm{BCB}-$ and control immature oocytes or in $\mathrm{BCB}+, \mathrm{BCB}-$, and control mature oocytes suggested a lack of interaction between G6PDH activity and the expression of these apoptosis-related proteins (Opiela et al., 2008). Moreover, Pawlak found that $\mathrm{BCB}+$ oocytes derived from ovaries of cycling gilts were more often apoptotic than $\mathrm{BCB}+$ oocytes derived from ovaries of pre-pubertal pigs (Pawlak et al., 2011a). We found a similar incidence of apoptosis in $\mathrm{BCB}+$ and control blastocyst groups in terms of DNA fragmentation measured

Table 1. Effect of G6PDH activity on the development of PA embryos

\begin{tabular}{lccc}
\hline Type of PA embryos & No. of mature oocytes used & $\begin{array}{c}\text { No. of cleaved embryos } \\
\text { cultured for } 48 \mathrm{~h}(\% \pm \mathrm{SE})\end{array}$ & $\begin{array}{c}\text { No. of blastocysts } \\
(\% \pm \mathrm{SE})\end{array}$ \\
\hline PA embryo derived from BCB+ oocytes & 119 & $96(80.7 \pm 1.6)^{\mathrm{a}}$ & $44(37.0 \pm 1.8)^{\mathrm{a}}$ \\
PA embryo derived from BCB- oocytes & 120 & $93(77.5 \pm 2.5)^{\mathrm{a}}$ & $16(13.3 \pm 1.4)^{\mathrm{b}}$ \\
Control PA embryos & 120 & $96(80.0 \pm 2.5)^{\mathrm{a}}$ & $43(35.8 \pm 1.4)^{\mathrm{a}}$ \\
\hline
\end{tabular}

G6PDH, glucose-6-phosphate dehydrogenase; PA, parthenogenetically activated; SE, standard error; BCB, brilliant cresyl blue.

Values in the same column with different small superscripts differ significantly $(\mathrm{p}<0.05)$. The experiment was repeated for three times. 


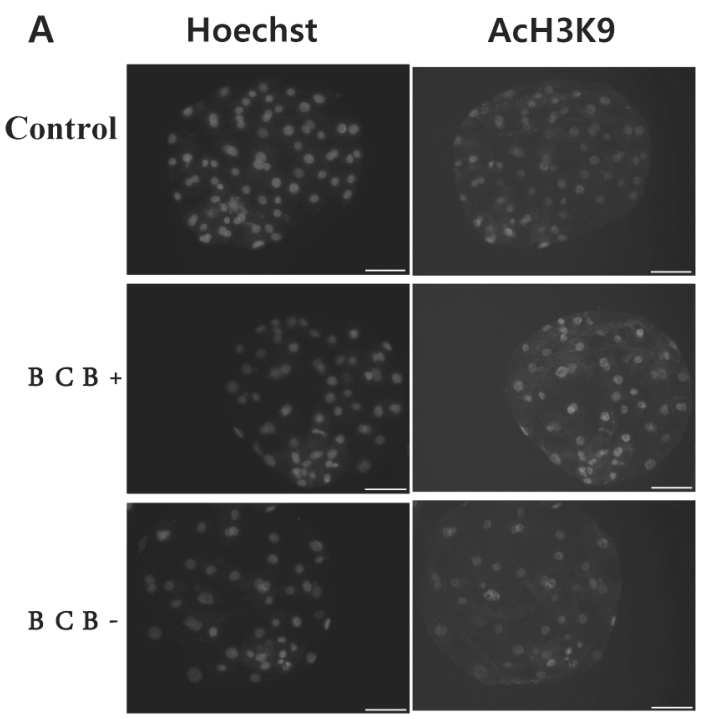

\section{B}

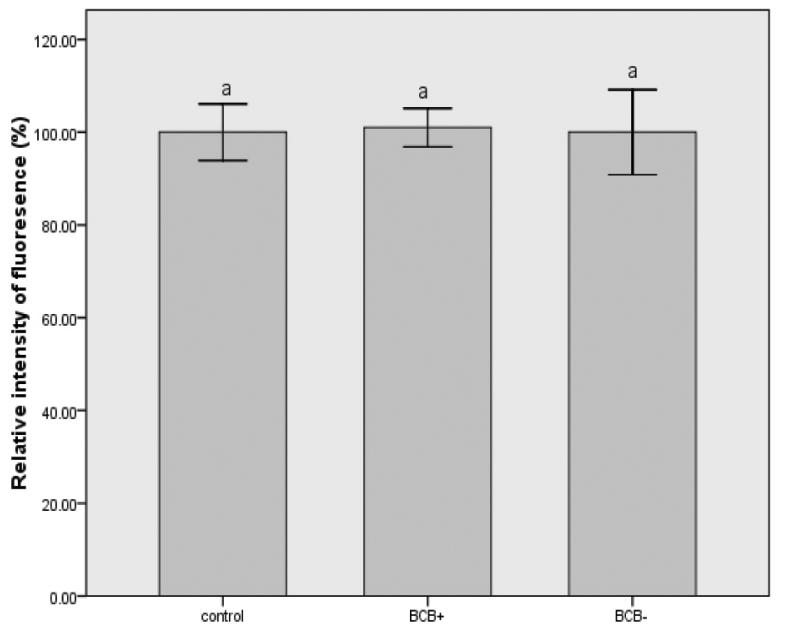

Figure 9. The acetylation levels of H3K9 (lysine 9 of histone H3) in blastocysts. (A) Staining of acetylated lysine 9 of histone H3 (AcH3K9) in control, brilliant cresyl blue positive $(\mathrm{BCB}+), \mathrm{BCB}-$ groups at blastocyst stage was shown. Each sample was counterstained with Hoechst 33342 to visualize DNA (blue). Bar $=50 \mu \mathrm{m}$. (B) Quantification of AcH3K9 signal intensities in BCB+ and $\mathrm{BCB}-$ at blastocyst stage was expressed relative to that of the control blastocysts (set as $100 \%$ ). The experiments were replicated three times. In each replication, $\mathrm{n}=15$ per group.

by the TUNEL assay. These phenomena might arise from donor age, or the apoptotic changes might be a reflection of a better adaptability to potentially harmful conditions during prolonged BCB staining (Anguita et al., 2009; Pawlak et al., 2011a).

The value of the $\mathrm{BCB}$ staining test for assessing oocyte quality seems to be questionable according to AcH3K9 levels and apoptotic features. Although this study indicated that G6PDH activity in porcine oocytes was associated with several subcellular characteristics such as GV chromatin configuration, $\mathrm{CG}$ migration, and mitochondrial distribution, other factors including the AcH3K9 level and apoptosis rates were not. Our results show a high level of heterogeneity in $\mathrm{BCB}+$ porcine oocytes in terms of the acetylation level of $\mathrm{H} 3 \mathrm{~K} 9$ and apoptotic traits. For future use, adding inhibitors of histone deacetylases or apoptosis

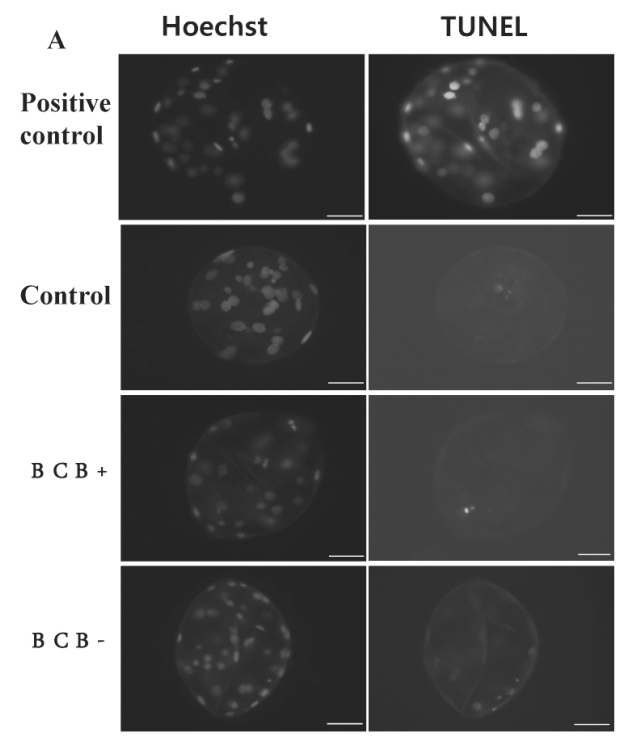

B

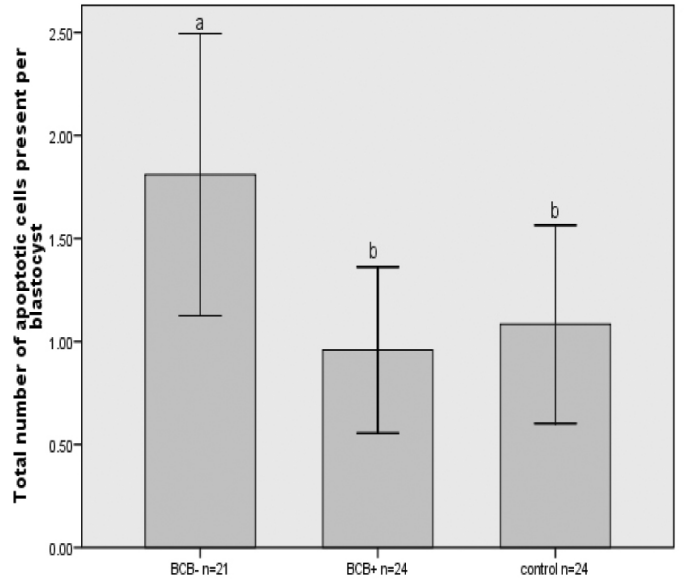

Figure 10. Incidence of apoptosis in blastocysts. (A) Representative photographs of terminal deoxynucleotidyl transferase nick-end labeling (TUNEL) assay of blastocysts (green). Each sample was counterstained with Hoechst 33342 to visualize DNA (blue). Bar $=50$ $\mu \mathrm{m}$. (B) Number of apoptotic cells in each blastocyst. Values with different superscripts differ significantly $(\mathrm{p}<0.05)$. BCB, brilliant cresyl blue. 
to the culture medium used for IVM of $\mathrm{BCB}+$ oocytes might improve the efficiency of the system.

\section{CONFLICT OF INTEREST}

We certify that there is no conflict of interest with any financial organization regarding the material discussed in the manuscript.

\section{ACKNOWLEDGMENTS}

This work was partly supported by National Natural Science Foundation of China (No. 31201804, No. 31101700), Natural Science Foundation of HLJ province (No. QC2013C021), Postdoctoral Programme of HLJ province (No. LBr-211032) and Heilongjiang Academy of Agricultural Sciences Doctoral scientific research projects (201507-32).

\section{REFERENCES}

Alm, H., H. Torner, B. Lohrke, T. Viergutz, I. M. Ghoneim, and W. Kanitz. 2005. Bovine blastocyst development rate in vitro is influenced by selection of oocytes by brillant cresyl blue staining before IVM as indicator for glucose-6-phosphate dehydrogenase activity. Theriogenology 63:2194-2205.

Anguita, B., M. T. Paramio, R. Morato, R. Romaguera, A. R. Jimenez-Macedo, T. Mogas, and D. Izquierdo. 2009. Effect of the apoptosis rate observed in oocytes and cumulus cells on embryo development in prepubertal goats. Anim. Reprod. Sci. 116:95-106

Bhojwani, S., H. Alm, H. Torner, W. Kanitz, and R. Poehland. 2007. Selection of developmentally competent oocytes through brilliant cresyl blue stain enhances blastocyst development rate after bovine nuclear transfer. Theriogenology 67:341-345.

Dai, X., J. Hao, X. J. Hou, T. Hai, Y. Fan, Y. Yu, A. Jouneau, L. Wang, and Q. Zhou. 2010. Somatic nucleus reprogramming is significantly improved by m-carboxycinnamic acid bishydroxamide, a histone deacetylase inhibitor. J. Biol. Chem. 285:31002-31010.

Das, Z. C., M. K. Gupta, S. J. Uhm, and H. T. Lee. 2010. Increasing histone acetylation of cloned embryos, but not donor cells, by sodium butyrate improves their in vitro development in pigs. Cell. Reprogram. 12:95-104.

Ericsson, S. A., M. L. Boice, H. Funahashi, and B. N. Day. 1993. Assessment of porcine oocytes using brilliant cresyl blue. Theriogenology 39:214

Guthrie, H. D. and W. M. Garrett. 2000. Changes in porcine oocyte germinal vesicle development as follicles approach preovulatory maturity. Theriogenology 54:389-399.

Ishizaki, C., H. Watanabe, M. M. Bhuiyan, and Y. Fukui. 2009. Developmental competence of porcine oocytes selected by brilliant cresyl blue and matured individually in a chemically defined culture medium. Theriogenology 72:72-80.

Kempisty, B., M. Jackowska, H. Piotrowska, P. Antosik, M. Wozna, D. Bukowska, K. P. Brussow, and J. M. Jaskowski. 2011. Zona pellucida glycoprotein 3 (pZP3) and integrin beta2
(ITGB2) mRNA and protein expression in porcine oocytes after single and double exposure to brilliant cresyl blue test. Theriogenology 75:1525-1535.

Mirshamsi, S. M., H. Karamishabankareh, M. Ahmadi-Hamedani, L. Soltani, H. Hajarian, and A. R. Abdolmohammadi. 2013. Combination of oocyte and zygote selection by brilliant cresyl blue (BCB) test enhanced prediction of developmental potential to the blastocyst in cattle. Anim. Reprod. Sci. 136:245-251.

Opiela, J. and L. Kątska-Książkiewicz. 2013. The utility of brilliant cresyl blue (BCB) staining of mammalian oocytes used for in vitro embryo production (IVP). Reprod. Biol. 13:177-183.

Opiela, J., L. Kątska-Książkiewicz, D. Lipinski, R. Slomski, M. Bzowska, and B. Rynska. 2008. Interactions among activity of glucose-6-phosphate dehydrogenase in immature oocytes, expression of apoptosis-related genes $\mathrm{Bcl}-2$ and $\mathrm{Bax}$, and developmental competence following IVP in cattle. Theriogenology 69:546-555.

Pawlak, P., E. Pers-Kamczyc, N. Renska, S. Kubickova, and D. Lechniak. 2011a. Disturbances of nuclear maturation in BCB positive oocytes collected from peri-pubertal gilts. Theriogenology 75:832-840.

Pawlak, P., N. Renska, E. Pers-Kamczyc, E. Warzych, and D. Lechniak. 2011b. The quality of porcine oocytes is affected by sexual maturity of the donor gilt. Reprod. Biol. 11:1-18.

Pawlak, P., E. Warzych, A. Chabowska, and D. Lechniak. 2014. Differences in cytoplasmic maturation between the $\mathrm{BCB}+$ and control porcine oocytes do not justify application of the $\mathrm{BCB}$ test for a standard IVM protocol. J. Reprod. Dev. 60:28-36.

Pereira, G. R., P. L. Lorenzo, G. F. Carneiro, S. Bilodeau-Goeseels, J. P. Kastelic, A. Esteller-Vico, M. Lopez-Bejar, and I. K. Liu. 2014. Selection of developmentally competent immature equine oocytes with brilliant cresyl blue stain prior to in vitro maturation with equine growth hormone. Zygote 22:500-504.

Pujol, M., M. López-Béjar, and M. T. Paramio. 2004. Developmental competence of heifer oocytes selected using the brilliant cresyl blue (BCB) test. Theriogenology 61:735744

Rodrigues, B. A., P. Rodriguez, A. E. Silva, L. F. Cavalcante, C. Feltrin, and J. L. Rodrigues. 2009. Preliminary study in immature canine oocytes stained with brilliant cresyl blue and obtained from bitches with low and high progesterone serum profiles. Reprod. Domest. Anim. 44 (Suppl 2):255-258.

Rodríguez-González, E., M. López-Bejar, D. Izquierdo, and M. T. Paramio. 2003. Developmental competence of prepubertal goat oocytes selected with brilliant cresyl blue and matured with cysteamine supplementation. Reprod. Nutr. Dev. 43:179-187.

Rodríguez-González, E., M. López-Bejar, E. Velilla, and M. T. Paramio. 2002. Selection of prepubertal goat oocytes using the brilliant cresyl blue test. Theriogenology 57:1397-1409.

Silva, D. S., P. Rodriguez, A. Galuppo, N. S. Arruda, and J. L. Rodrigues. 2013. Selection of bovine oocytes by brilliant cresyl blue staining: effect on meiosis progression, organelle distribution and embryo development. Zygote 21:250-255.

Su, J., Y. Wang, R. Li, H. Peng, S. Hua, Q. Li, F. Quan, Z. Guo, and Y. Zhang. 2012. Oocytes selected using BCB staining enhance nuclear reprogramming and the in vivo development of SCNT embryos in cattle. PloS one 7:e36181. 
Sun, Q. Y., G. M. Wu, L. Lai, K. W. Park, R. Cabot, H. T. Cheong, B. N. Day, R. S. Prather, and H. Schatten. 2001. Translocation of active mitochondria during pig oocyte maturation, fertilization and early embryo development in vitro. Reproduction 122:155-163.

Sun, X. S., Y. Liu, K. Z. Yue, S. F. Ma, and J. H. Tan. 2004. Changes in germinal vesicle $(\mathrm{GV})$ chromatin configurations during growth and maturation of porcine oocytes. Mol. Reprod. Dev. 69:228-234
Tiffin, G. J., D. Rieger, K. J. Betteridge, B. R. Yadav, and W. A. King. 1991. Glucose and glutamine metabolism in preattachment cattle embryos in relation to sex and stage of development. J. Reprod. Fertil. 93:125-132.

Wu, Y. G., Y. Liu, P. Zhou, G. C. Lan, D. Han, D. Q. Miao, and J. H. Tan. 2007. Selection of oocytes for in vitro maturation by brilliant cresyl blue staining: A study using the mouse model. Cell Res. 17:722-731.

Yang, C. R., D. Q. Miao, Q. H. Zhang, L. Guo, J. S. Tong, Y. Wei, X. Huang, Y. Hou, H. Schatten, Z. Liu, and Q. Y. Sun. 2010. Short-term preservation of porcine oocytes in ambient temperature: novel approaches. PloS one 5:e14242. 